\title{
A CALORIMETRIC METHOD FOR DETERMINING THE INTRINSIC ENERGY OF A GAS AS A FUNCTION OF THE PRESSURE
}

\author{
By Edward W. Washburn
}

\begin{abstract}
If a known mass of gas compressed in a bomb at a known pressure be immersed in a calorimeter and allowed to expand slowly to atmospheric pressure, the cooling effect can be compensated by electrical heating. Since the external work is accurately known, a precision discussion shows that the method should yield an accurate value ( $\pm<1$ cal. for a liter bomb) for the accompanying change, $\Delta U$, in the internal or intrinsic energy of the gas. In order to obtain reliable results, the energy effects associated with the changes in stress on the walls of the bomb must be corrected for or reduced to a negligible amount by proper bomb design. From the standpoint of simplicity, rapidity, and accuracy the method is probably superior to any other means of determing this quantity.
\end{abstract}

ABSTRACT

CONTENTS

I. Nomenclature

II. Introduction

III. Methods of evaluating $U_{T}=f(p)$

1. The $p-v-T$ method.

2. The adiabatic Joule-Thomson method

3. The isothermal Joule-Thomson method $\ldots \ldots$

4. The direct calorimetric method

IV. The calorimetric technic

$V$. The energy content of the bomb as a function of the pressure...

VI. Effects of adsorption

VII. The temperature coefficient of $U_{T}$

VIII. Precision discussion

\section{NOMENCLATURE}

$B$ Barometric pressure.

$C_{p}$ Heat capacity at constant pressure.

j Joule.

M Molecular weight.

$m$ Mass.

$p$ Pressure.

$Q$ Quantity of heat.

$R$ Gas constant per mole.

$T$ Absolute temperature.

$t$ Centigrade temperature.

$U$ Intrinsic energy.

$v$ Volume.

$W$ Work.

$\alpha$ Linear coefficient of thermal expansion.

$\mu$ Joule-Thomson coefficient. 


\section{INTRODUCTION}

For any system whose thermodynamic condition is determined solely by temperature and pressure, the first law of thermodynamics assumes the form

$$
d U=d Q-p d v
$$

in which $U$ is the so-called "total," "internal," or "intrinsic" energy of the system, $Q$ is the heat added to it, $p$ is the pressure upon it, and $v$ is its volume.

For any elastic system the above relation when combined with the second law yields the familiar thermodynamic equation

$$
-\left(\frac{\partial U}{\partial p}\right)_{T}=p\left(\frac{\partial v}{\partial p}\right)_{T}+T\left(\frac{\partial v}{\partial T}\right)_{p}
$$

in which $T$ is the absolute temperature.

The integral of this equation, $U_{T}=f(p)$ for a gaseous system at the temperature $T$, is the quantity sought. Aside from its general thermodynamic importance this quantity is of practical interest in connection with bomb calorimetry where a knowledge of its value for oxygen and for mixtures of oxygen with carbon dioxide is needed in order to evaluate the changes in the heat content of these gases with the pressure.

\section{METHODS OF EVALUATING $U_{T}=f(p)$}

\section{THE $p^{-V-T}$ METHOD}

By operating upon an appropriate equation of state, the righthand member of equation (2) can be obtained as a pressure function with numerical coefficients and the equation can then be integrated. In practice this method suffers from the disadvantage that there are very few gases for which $p-v-T$ data are known with sufficient accuracy, and the acquisition of such data is a difficult and tedious undertaking.

In the absence of more exact information, it is however, convenient to remember that as a first approximation for moderate pressures the following equation, obtained by operating upon the BeattieBridgeman equation of state, ${ }^{1}$ may be employed

$$
-\Delta U]_{0}^{p}=\left(\frac{A_{0}}{R T}+\frac{3 c}{T^{3}}\right) p
$$

in which $A_{0}$ and $c$ are constants appearing in the equation of state.

\section{THE ADIABATIC JOULE-THOMSON METHOD}

In this method $\left[\frac{\partial(p v)}{\partial p}\right]_{T}$ is evaluated as a pressure function with the aid of measured $p v$ values at the temperature $T$ or, failing these, with the aid of an equation of state, and $\left(\frac{\partial U}{\partial p}\right)_{T}$ is obtained from the thermodynamic relation

$$
-\left(\frac{\partial U}{\partial p}\right)_{T}=\mu C_{p}+\left[\frac{\partial(p v)}{\partial p}\right]_{T}
$$

1 J. A. Beattie, Phys. Rev., vol. 32, p. 699, 1928. 
in which $\mu$ is the Joule-Thomson coeffieient and $C_{p}$ the heat capacity at constant pressure. Both $\mu$ and $C_{p}$ are pressure functions.

This method also suffers from the disadvantage that reliable $\mu$ values are available only for two or three gases and the technic of obtaining them is exceedingly difficult. From the standpoint of attainable accuracy, however, the method is perhaps superior to the $p-v-T$ method, especially at temperatures remote from room temperature.

\section{THE ISOTHERMAL JOULE-THOMSON METHOD}

This method differs from the preceding one only in that the product, $\mu C_{p}$, is determined calorimetrically by supplying measured electrical energy at such a rate as to compensate for the temperature drop which occurs in the classical Joule-Thomson experiment. The experimental technic of this "isothermal porous-plug experiment" has only recently been developed to the point where the method gives promise of yielding valuable and reliable results. ${ }^{2}$

\section{THE DIRECT CALORIMETRIC METHOD}

Since $\Delta U$ is an energy quantity, the method of measuring it directly with the aid of a calorimeter would at first sight appear to be the most convenient and accurate one to employ. With the exceptions noted below, however, attempts to employ this method have failed to give results of sufficient accuracy. The chief difficulty appears to arise from the energy effects associated with the changes in stress on the walls of the containing vessel.

One simple method of carrying out the calorimetric determination is to determine

$$
\left.Q=\int_{p}^{B}\left(\frac{\partial U}{\partial p}\right)_{T} d p+W=\Delta U\right]_{p}^{B}+B \Delta v
$$

where $B \Delta v$ is the work done against the atmosphere when the gas contained in a bomb is allowed to expand isothermally from the initial pressure $p$ to the barometric pressure $B$, or in general to any low pressure $P$ such that for all lower pressures the integral $\int_{p}^{O}\left(\frac{\partial U}{\partial p}\right)_{T} d p$ is calculable or is negligibly small.

In this form the method has been used by Douglas ${ }^{3}$ to determine the total energy change accompanying the evaporation of a liquefied gas, and by Bennewitz and Andreewa ${ }^{4}$ to determine $U$ as a function of $p$ for gases in the neighborhood of the critical point. In both of these investigations the value of $\Delta U$ was a comparatively large quantity, and great refinement of calorimetric technic was not required in order to obtain a fair percentage accuracy. For temperatures far above the critical temperature, however, $\Delta U$ is a small quantity, calorimetric technic of high precision is required and the contribution of the containing vessel to the total heat effect can not be ignored.

2 See (a) F. G. Keyes and S. C. Collins, Proc. Nat. Acad. Sci., vol. 18, p. 328, 1932; (b) A. Eucken, K. Clusius, and W. Berger, Z. tech. Phyisick, vol. 13, p. 267, 1932."

3 Douglas, Phil. Mag., vol. 18, p. 159, 1909.

Bennewitz and Andreewa, Z. physik. Chem., vol. 142A, p. 37, 1929. 


\section{THE CALORIMETRIC TECHNIC}

The gas to be investigated, compressed into a suitable bomb, is immersed in a constant temperature bath at the temperature $T$. When temperature equilibrium has been established, the pressure $p$ in the bomb is measured, the valve is closed, the bomb is removed from the bath and weighed to determine the mass of the contained gas. The bomb is then transferred to the calorimeter and its outlet connected to a long coil of metal tubing which is likewise immersed in the calorimeter. When equilibrium has been established in the calorimeter, observations are started and the time-temperature foreperiod accurately established. The valve of the bomb is now opened gradually and the gas escapes through the long coil, which is so designed as to bring the gas to the calorimeter temperature and to barometric pressure before it escapes into the atmosphere. Coincident with this operation and throughout its course, measured electrical energy is added to the calorimeter at such a rate as to maintain the temperature constant. When the pressure in the bomb has fallen to atmospheric, the electrical heat is cut off and the calorimetric after-period is accurately determined. The heat, $Q$, of the process is now calculable as is also the work done against the atmosphere, and from equation (5) the quantity $\Delta U]_{p}^{B}$ is then obtained.

The contribution of the bomb itself to the observed heat effect can, for a properly designed bomb, be computed as explained below or it may be eliminated by standardizing the operation with a gas for which accurate $\Delta U$ values are available.

\section{THE ENERGY CONTENT OF THE BOMB AS A FUNCTION OF THE PRESSURE}

The bomb itself is an elastic system and its intrinsic energy changes with the tension on its walls. Unlike the gas, however, it may not return promptly to its original energy state on release of the tension, but instead may exhibit a lag.

For this reason it is desirable (1) to design a bomb for which the change of intrinsic energy with pressure shall be as small as possible, and (2) to suitably anneal the bomb and to age it by subjecting it to a number of cycles of extension and contraction by repeated filling and emptying with compressed gas.

Since the bomb is itself an elastic system the following thermodynamic relation analogous to equation (2) is applicable

$$
\left(\frac{\partial U}{\partial p_{b}}\right)_{T}=p_{b}\left(\frac{\partial v_{i}}{\partial p_{b}}\right)_{T}-B\left(\frac{\partial v_{e}}{\partial p_{b}}\right)_{T}+T\left(\frac{\partial v_{i}}{\partial T}\right)_{p_{b}}
$$

in which $p_{b}$ is the internal pressure, $v_{t}$ the internal volume, $B$ the constant external barometric pressure, and $v_{e}$ the exterior or bulk volume. Assuming the approximations

$$
\left(\frac{\partial v_{i}}{\partial p_{b}}\right)_{T}=\left(\frac{\partial v_{e}}{\partial p_{b}}\right)_{T} \text { and } \frac{1}{v_{i}}\left(\frac{\partial v_{i}}{\partial T}\right)_{p_{b}}=3 \alpha
$$

both independent of the pressure, and integrating gives

$$
\Delta U]_{B}^{p_{b}}=\frac{\left(p_{b}-B\right)^{2}}{2}\left(\frac{\partial v_{i}}{\partial p_{b}}\right)_{T}+3 \alpha v_{i} T\left(p_{b}-B\right)
$$


in which $\alpha$ is the linear coefficient of thermal expansion of the material composing the bomb.

For a cylindrical steel bomb with hemispherical ends, a wall thick-

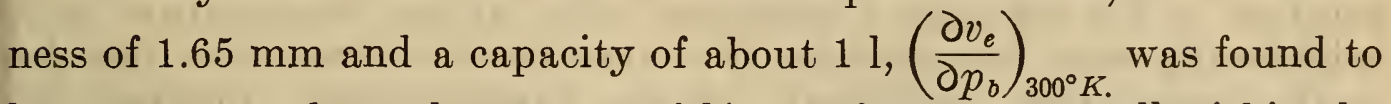
be constant and equal to $39 \times 10^{-6} \mathrm{l} / \mathrm{atm}$., for stresses well within the elastic limit.

For $p_{b}=50 \mathrm{~atm}$. and $B=1 \mathrm{~atm}$. we have therefore at $300^{\circ} \mathrm{K}$.

$$
\begin{aligned}
\Delta U]_{1}^{50} & =\frac{49^{2}}{2} \times 39 \times 10^{-6}+3 \times 11 \times 10^{-6} \times 1 \times 300 \times 49 \text { l-atm } . \\
& =4.74+49.16=53.9 \mathrm{j}
\end{aligned}
$$

If the contained gas is air at 50 atm. it will give $-\Delta U]_{1}^{50}=560 \mathrm{j}$ approximately. The contribution of the bomb therefore amounts to about 10 per cent of intrinsic energy change of the gas and, if the bomb is slow in returning to its original energy state, its contribution can not in practice be calculated with sufficient accuracy.

Now the first term in equation (9) is a work term, and the quantity $\left(\frac{\partial v_{e}}{\partial p_{b}}\right)_{T}$ can be directly measured with sufficient accuracy by immersing the filled bomb in a constant-temperature volumeter and releasing the pressure. For a steel bomb no appreciable lag is observed in this quantity. The lag therefore affects only the second term which is the larger of the two. This term can be materially reduced by constructing the bomb of a material having a low coefficient of thermal expansion. Thus if a suitable invar steel is selected for this purpose, equation (9) for the same conditions becomes

$$
\Delta U\left[{ }_{1}^{50}=4.74+4.92=9.7 \mathrm{j}\right.
$$

The total bomb contribution will now be only 1.7 per cent of the gas effect and that part of it which is affected by lag will be less than 1 per cent and the uncertainty arising from the lag will be a negligible quantity in comparison with the other errors of measurement.

\section{EFFECTS OF ADSORPTION}

In all experimental work involving $p-v-T$ or energy relations of gases the influences of adsorbed gas on the walls of the containing vessel must be considered. These influences are usually measured and eliminated by a series of experiments with different areas of exposed surface in contact with the gas. In the present instance such experiments can be readily made by placing wire or foil (of the same material as the bomb) inside the bomb in order to increase the area of metal surface exposed. Since, however, in the present method the pressure of the gas does not fall below atmospheric during the experiment, the adsorbed gas which leaves the walls of the bomb is only the small and probably negligible amount which becomes adsorbed when the pressure is raised from 1 atmosphere to the initial pressure $p_{b}$. 


\section{THE TEMPERATURE COEFFICIENT OF $U_{T}$}

For correcting the calorimetric determinations to a common temperature or for reducing a measured value at one temperature to a neighboring other temperature, it is convenient to remember that the small temperature coefficient can be approximately obtained by differentiating equation (3) which gives

$$
\frac{100 d(\Delta U)}{\Delta U d t}=\frac{-100}{T}\left[1+6 c T^{3}\left(\frac{A_{0}}{R T}+\frac{3 c}{T^{3}}\right)\right]
$$

Thus for $\mathrm{O}_{2}$ at $25^{\circ} \mathrm{C}$. this equation gives 0.4 per cent per degree.

\section{PRECISION DISCUSSION}

In equation (5) the work done by the expanding gas will be

$$
W=\mathrm{B} \Delta v=B v-B v i=\frac{m}{M} R T\left(1+\beta_{T} B\right)-B v_{i}
$$

and equation (5) may be written

$$
Q=m\left[f(p)+\frac{R T\left(1+\beta_{T} B\right)}{M}\right]-B v_{i}
$$

in which $m$ is the mass of gas contained in the bomb of volume $v_{i}$ at the pressure $p$ and temperature $T, R$ is the gas constant, $M$ the molecular weight of the gas, $\beta_{T}$ is a small coefficient expressing the deviation from the perfect gas law at atmospheric pressure and $T^{\circ} \mathrm{K}, B$ is the prevailing barometric pressure, and $f(p)$ is the value of $\Delta U_{p}^{B}$ for $1 \mathrm{~g}$ of the gas at $T^{\circ} \mathrm{K}$.

Using the value of $\Delta U]_{p}^{1}=f(p)$ for air, one can compute the precision required in each measured quantity in order to obtain a desired precision in the quantity $\Delta U$ for this gas. If we take $t=25^{\circ} \mathrm{C}$., $v_{i}=1$ liter and assume an allowable error of 0.25 cal. from each measured quantity, we obtain the results shown in the following table. 
TABLE 1.-Error in each measured quantity which produces an error of $0.25 \mathrm{cal}$. in the quantity $\Delta U]_{p}^{1}$

\begin{tabular}{|c|c|c|c|c|c|}
\hline & $\Delta U]_{p \text { atm. }}^{1 \text { atm }}$ (air at $25^{\circ} \mathrm{C}$.), cal. & 22.0 & 50.0 & 89.2 & 139 \\
\hline & a d & $\begin{array}{l}20 \\
0.22\end{array}$ & $\begin{array}{l}30 \\
0.14\end{array}$ & $\begin{array}{l}40 \\
0.11\end{array}$ & $\begin{array}{l}50 \\
0.09\end{array}$ \\
\hline & $\left\{\begin{array}{l}v_{i}-1 \\
\delta_{i}-1-2\end{array}\right.$ & $\begin{array}{r}1 \\
10\end{array}$ & $\begin{array}{r}1 \\
10\end{array}$ & $\begin{array}{r}1 \\
10\end{array}$ & $\begin{array}{r}1 \\
10\end{array}$ \\
\hline & $\left\{\begin{array}{l}m \\
\delta m_{-}\end{array}\right.$ & $\begin{array}{l}24 \\
11\end{array}$ & $\begin{array}{l}36 \\
11\end{array}$ & $\begin{array}{l}48 \\
11\end{array}$ & $\begin{array}{l}60 \\
11\end{array}$ \\
\hline & $\left\{B_{1} B_{-}-m_{1}\right.$ & $\begin{array}{l}760 \\
8.2\end{array}$ & $\begin{array}{l}760 \\
8.4\end{array}$ & $\begin{array}{l}760 \\
8.6\end{array}$ & $\begin{array}{l}760 \\
9.1\end{array}$ \\
\hline & $\left\{\begin{array}{l}\text { Electrical energy equivalent } \\
\delta t \\
\delta t^{\prime}-\end{array}\right.$ & $\begin{array}{r}485 \\
0.0036 \\
\quad .0001\end{array}$ & $\begin{array}{r}757 \\
0.0036 \\
.0001\end{array}$ & $\begin{array}{r}1,048 \\
0.0036 \\
.0001\end{array}$ & $\begin{array}{r}1,348 \\
0.0036 \\
.0001\end{array}$ \\
\hline & $\left\{\begin{array}{l}\text { Stirring energy } \\
100 \delta \text { (S. E.) } /(\mathrm{S} . \mathrm{E} .)\end{array}\right.$ & $\begin{array}{l}10 \\
2.5\end{array}$ & $\begin{array}{l}10 \\
2.5\end{array}$ & $\begin{array}{l}10 \\
2.5\end{array}$ & $\begin{array}{l}10 \\
2.5\end{array}$ \\
\hline & $\left\{\begin{array}{l}T \\
{ }_{\delta} \\
T_{-}\end{array}\right.$ & ${ }^{298} .16$ & ${ }^{298} .11$ & ${ }^{298} .08$ & ${ }^{298} .06$ \\
\hline & $\left\{\begin{array}{l}\beta_{T}+\ldots \\
\delta_{T} \beta_{2}\end{array}\right.$ & .00037 & .00037 & $\begin{array}{l}.00037 \\
.0003\end{array}$ & $\begin{array}{l}.00037 \\
.0002\end{array}$ \\
\hline
\end{tabular}

Of the items shown in the table, Nos. $2,4,7$, and 8 can be eliminated as sources of error since they can obviously be easily measured with much more than the required accuracy.

Item 1.- For the pressure measurements a good calibrated Bourdon gage could be employed for pressures up to 50 atm. At higher pressures a more accurate method of measuring the pressure would be required.

Item 3.- In the mass determination, somewhat more than the required accuracy can be easily obtained with a liter bomb and with a balance sensitive to a few milligrams under loads up to $2 \mathrm{~kg}$.

Item 5.- In determining the electrical energy required to equalize the cooling effect of the expanding gas no significant errors will be made in measuring the actual energy input employed. The total error from this source will be determined by the accuracy of the temperature control during the experiment and the accuracy in determining the small difference between the initial and final equilibrium temperatures. The value of $\delta t$ shown in the table represents the permissible integrated average temperature difference between the calorimeter and the jacket during the experiment, assuming a reaction time of 10 minutes and an over-all heat-transfer coefficient of $7.2 \mathrm{cal} . \mathrm{min} .^{-1}{ }^{\circ} \mathrm{C}^{-1}$. The value $\delta t^{\prime}$ represents the accuracy with which the difference between the initial and final temperatures must be known with a calorimeter having a water equivalent of 2,500 g.

Item 6.-An efficient stirrer will supply energy to the calorimeter at the rate of about 1 cal. per minute. For an allowable error of not more than $0.25 \mathrm{cal}$. from this source it is obvious that throughout the period of the experiment ( 30 minutes) the stirring power should be constant within \pm 2.5 per cent.

From the foregoing discussion it appears that it should be possible without much difficulty, to determine the quantity $\Delta U]_{p}^{1}$ for 1 liter of

5 The necessity of employing a pressure gauge can be dispensed with, if desired, by the use of twin bombs. See Washburn, B. S. Jour. Research, vol. 9, p. 271, 1932. 
gas with an error of less than 1 cal. A forthcoming paper by Rossini and Frandsen describes some experimental results obtained by the method.

In conclusion, it may be noted that by combining the calorimetric value for $\left(\frac{\partial U}{\partial p}\right)_{T}$ with the calorimetric value for $\mu C_{\mathfrak{p}}$, both $p\left(\frac{\partial v}{\partial p}\right)_{T}$ and $T\left(\frac{\partial v}{\partial T}\right)_{p}$ can be directly obtained, if the volume is known, and this is probably the most accurate method of determining these two coefficients.

Washington, August 3, 1932. 